\title{
PHYTOCHEMICAL EVALUATION AND FUNCTIONAL GROUP DETECTION OF ETHANOLIC LEAF AND ROOT EXTRACTS OF Datura metel
}

\author{
Chieme Sunday Chukwudoruo $^{1 *}$, Stanley Chukwuma Okereke ${ }^{2}$, \\ Chinyere Henrietta Onuoha ${ }^{1}$, Olachi Lilian Osuagwu', \\ Chioma Blessing Onowoh ${ }^{1}$ and Favour Ntite Ujowundu ${ }^{1}$ \\ ${ }^{1}$ Department of Biochemistry, Federal University of Technology, Owerri \\ ${ }^{2}$ Department of Biochemistry, Abia State University, Uturu \\ *Corresponding Email: cchukwudoruo@gmail.com
}

Cite this article:

Chieme S.C., Stanley C.O., Chinyere H.O., Olachi L.O., Chioma B.O., Favour N.U. (2022), Phytochemical Evaluation and Functional Group Detection of Ethanolic Leaf and Root Extracts of datura metel. African Journal of Biology and Medical Research 5(1), 30-52. DOI: 10.52589/AJBMR96ZVVKMM

\section{Manuscript History \\ Received: 12 Oct 2021 \\ Accepted: 3 Nov 2021}

Published: 22 Feb 2022

Copyright $@ 2022$ The Author(s). This is an Open Access article distributed under the terms of Creative Commons AttributionNonCommercial-NoDerivatives 4.0 International (CC BY-NC-ND 4.0), which permits anyone to share, use, reproduce and redistribute in any medium, provided the original author and source are credited.
ABSTRACT: Datura metel is a plant with various medicinal parts. This work gears towards the phytochemical evaluation, identification and functional group detection of ethanolic leaf and roots extracts of Datura metel. Gas Chromatography-Mass spectrometry $(G C-M S)$ and Fourier -Transform Infrared Spectroscopy (FTIR) analytical instruments were used for studies. The preliminary phytochemical evaluation of plant parts revealed presence of alkaloids, flavonoids, saponins, tanins and terpenes. The chromatogram result for the Gas ChromatographyMass Spectroscopy (GC-MS), detected 30 bioactive compounds with high percentage composition and molecular weights. Thiophene, 2,3-dehydro, an isomer of dehydrothiophene $\left(\mathrm{C}_{4} \mathrm{H}_{6} \mathrm{HS}\right)$ had the least retention time and highest percentage composition of $38.914 \%$ for the root extracts while 2-methyl-3thiosemicarbazide $\left(\mathrm{C}_{2} \mathrm{H}_{7} \mathrm{~N}_{3} \mathrm{~S}\right)$ and Benzene hexanenitirle, dimethyl-E-oxo $\left(\mathrm{C}_{14} \mathrm{H}_{17} \mathrm{HNO}\right)$ both had the highest percentage composition for the leaf extracts. The chromatogram result for Fourier-transform infrared spectroscopy (FTIR) revealed that alkenes $(=\mathrm{C}-\mathrm{H})$ and carbon tetrachloride $\left(\mathrm{C}-\mathrm{CL}_{4}\right)$ had the highest and least wavelengths for both extracts respectively. The presence of bioactives detected and identified in this study, showed that plants may serve as reservoir for biologically active compounds and hence in addition to its medicinal values can also be used for diverse purposes in the industry.

KEYWORDS: Datura metel, Phytochemicals, FTIR, GC-MS, Functional group, Bioactives. 


\section{INTRODUCTION}

Nature has endowed us with so many botanical wealth which provides us with medicinal plants that has some potential and valuable effect in health care. Medicinal plants has been used for health purposes for several thousands of years (Chavhan etal.,2018), due to the presence of phytochemicals synthesized as secondary metabolites that helps to protect them from environmental damage and also contribute to their colour, aroma and flavor (Mattai,2010).

Datura metel is one of the most commonly used medicinal plant. It was first described by the scientist Linneaus in the year 1753 (Dixon and Jeena,2017). The plant is said to have originated from North America (Alabiri etal., 2013) and widely cultivated in all tropical and subtropical regions of the globe for its beautiful flowers (Monira and Munan,2012). It has numerous common names such as Jimson weed, Thorn apple, Devil apple, Angel trumpet (Jmdhade etal.,2010). Datura metel is about $1.5 \mathrm{~m}$ tall, its leaves are simply broad and dark green in colour. The fruits are like capsule covered with short spines or thorns (Monira and Munan,2012). According to several reports on preliminary phytochemical investigations, Datura metel, is found to be rich in some bioactive compounds like alkaloids, flavonoids, tanins, saponins. Scopolamines is its major bioactive components under category of alkaloids (Dixon and Jeena, 2017) and are responsible for its medicinal properties. Datura metel is used in treatment of some diseases like epilepsy, heart disease, asthma (Alabiri etal.,2013), in Nigeria. it also has a wide application in pharmacology as it is used in producing some medications used for wound healing, treatment of hairfall, dandruff, skin disorder, diarrhea, fever, pain etc. (Yang etal.,2014). Despite all these application, Datura metel also have intoxicating and hallucinogenic properties.

Gas Chromatography and Mass Spectometry (GC-MS) is mainly used in the separation and analysis of multi component mixtures such as essential oils, hydrocarbons and solvents (Kadhim etal. 2010). It is also used for quantitative and qualitative analysis of mixtures, purification of compounds and determination of thermochemical constants in solution and vapor pressure and activity coefficient. GC-MS analysis can identify nature of compounds even less than 1mg present in crude plant extract (Muthulaskshmi etal. 2012). Fourier transform Infrared Spectroscopy (FTIR) has been the most powerful tool for identifying the type of chemical bond or functional group present in analytes. It interprets its infrared absorption spectrum by varying observation in wavelength of the light absorbed which is the salient feature of the chemical bonds as seen in the annoted spectrum. (Coates, 2000)

It is important to isolate, identify and characterize, the bioactive present in these traditionally grown medicinal plants as well as attention given to harness other components beneficial for man such as industrially and agriculturally, hence the objective of this research work.

\section{MATERIALS AND METHODS}

\section{Collection and authentication of plant materials.}

The leaves and roots of datura metel were harvested in Federal University of Technology Owerri, Imo State. It was identified by a plant taxonomist, Dr D.I. Edet from department of Forestry and Wildlife, School of Agricultural and Agricultural Technology, Federal University 
of Technology Owerri. The plant was pressed by Dr, F.A. Faruwa, and deposited at the university herbarium with voucher number FUTO/FW/HERB/2019/052 for reference purpose.

\section{Preparation of plant extracts}

The plant leaf and root were detached, washed free of sand and debris, air-dried for about five weeks at room temperature, and pulverized using an electric blender. Solvent extracts of datura metel was prepared by weighing $50 \mathrm{~g}$ of powdered sample into $1000 \mathrm{ml}$ capacity beaker, then $500 \mathrm{ml}$ of ethanol added to each beaker containing the samples, the sample was thoroughly mixed and allowed to stand for 24 hours, then filtered, using muslin bag and whatmann filter paper. The sample was further concentrated using a water bath at a temperature of $35^{\circ} \mathrm{C}$, covered firm and refrigerated at temperature of $4^{\circ} \mathrm{C}$.

\section{Preliminary phytochemical screening}

Wagner reagent test was used in testing for alkaloids. From each of the extracts, $3 \mathrm{ml}$ filtrate was acidified in $1 \mathrm{ml}$ hydrochloric acid and few drops of wagner'sreagent added. A reddish brown precipitate indicated the presences of alkaloids. Lead Acetate was used to test for presence of flavonid. $1 \mathrm{ml}$ of each extracts was taken and lead acetate solution added, a yellow precipitate confirmed the presence of Flavonids. To $1 \mathrm{ml}$ of extracts, $20 \mathrm{ml}$ of distilled water was added and agitated in a graduated cylinder for 15 minutes. The formation of $1 \mathrm{~cm}$ layer of foam indicated presence of saponins, using the foam test. Terpenoids present was tested using salkwoski test, hence $5 \mathrm{ml}$ of each extract was added to $2 \mathrm{ml}$ of chloroform and $3 \mathrm{ml}$ of Con $\mathrm{H}_{2} \mathrm{SO}_{4}$ to form a layer of reddish brown coloration, its interface was said to form a positive result. Presence of tannins was determined using iron (iii) chloride solution $\left(\mathrm{FeCl}_{3}\right)$. Hence $5 \%$ $\mathrm{FeCl}_{3}$ solution was added to extract and a blue black colouration was formed.

\section{Structural elucidation of samples}

This was carried out using GC-MS procedure. Exact $2 \mathrm{~g}$ of extracted powder was weighed into an amber glass bottle and added $3 \mathrm{ml}$ ethanol. The sample was then shaken vigorously for 2 hours filtered and concentrated, $2 \mathrm{ml}$ of the extracted samples were then analyzed in GC column. This operation was carried out using GC (Agilent 6890N) and MS (5975B MSD). It was equipped with DB-5ms capillary columns $(30 \mathrm{~m}$ by $0.25 \mathrm{~m})$ film thickness $(0.25 \mu \mathrm{m})$ and temperature was set at $40^{\circ} \mathrm{C}$, then to $15^{\circ} \mathrm{C}$ and $230^{\circ} \mathrm{C}$ at the rate of $10^{\circ} \mathrm{C} / \mathrm{mins}$ which held for 5 minutes. Heluim served as gas carrier having a flu rate of $1 \mathrm{ml} / \mathrm{mins}$. Split rate and ionization voltage were $110 \mathrm{ev}$ and $70 \mathrm{ev}$ respectively. Phytochemicals were identified by mass spectra peak value which was compared with the database of National Institute of Science and 'Technology.

\section{Determination of functional bonds and chemical groups}

This was carried out using Fourier transform spectrometer (vectex 80, Bruker optics) equipped with an attenuated total reflectance (ATR) accessory (MIRracle, Pike technologies). The spectrum was recorded in the spectral range of $600 \mathrm{~cm}^{-1}$ to $4000 \mathrm{~cm}^{-1}$ at a resolution of $2 \mathrm{~cm}^{-}$ with a mercury cadmium tellun dedectector (MCT Dectector) (Coates, 2000). 


\section{RESULT}

The preliminary phytochemical screening for active compounds of ethanolic leaf and root extracts of Datura metel was qualitatively analyzed and the results listed in table 3.1. Then Table 3.2.and 3.3.Showed results from Fourier Transform Infrared Spectroscopy (FTIR) analysis revealing presence of alkenes, alkane, amine, alkyl halides and isothiocynate groups for both ethanolic extracts.

The Gas Chromatography Mass spectrometry (GC-MS) chromatogram for both extracts of Datura metel showed various peaks and indicated presence of 30 compounds as shown in tables 3.4 and 3.5. These compounds were identified and confirmed by interpretation of mass spectrum of GC-MS using the database of National Institute of Standards and Technology (NIST).

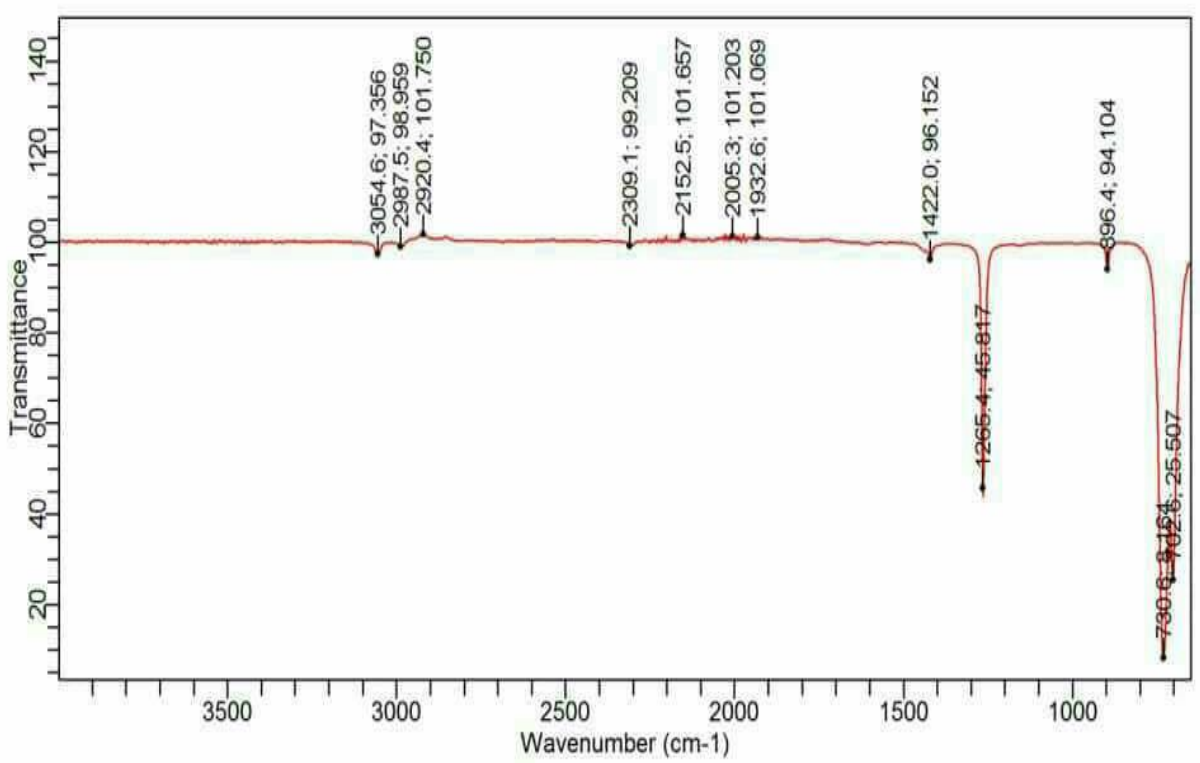

Fig 1.0. The chromatogram of the FTIR analyses of the ethanolic leaf extract of Datura metel 
African Journal of Biology and Medical Research

ISSN: 2689-534X

Volume 5, Issue 1, 2022 (pp. 30-52)

www.abjournals.org

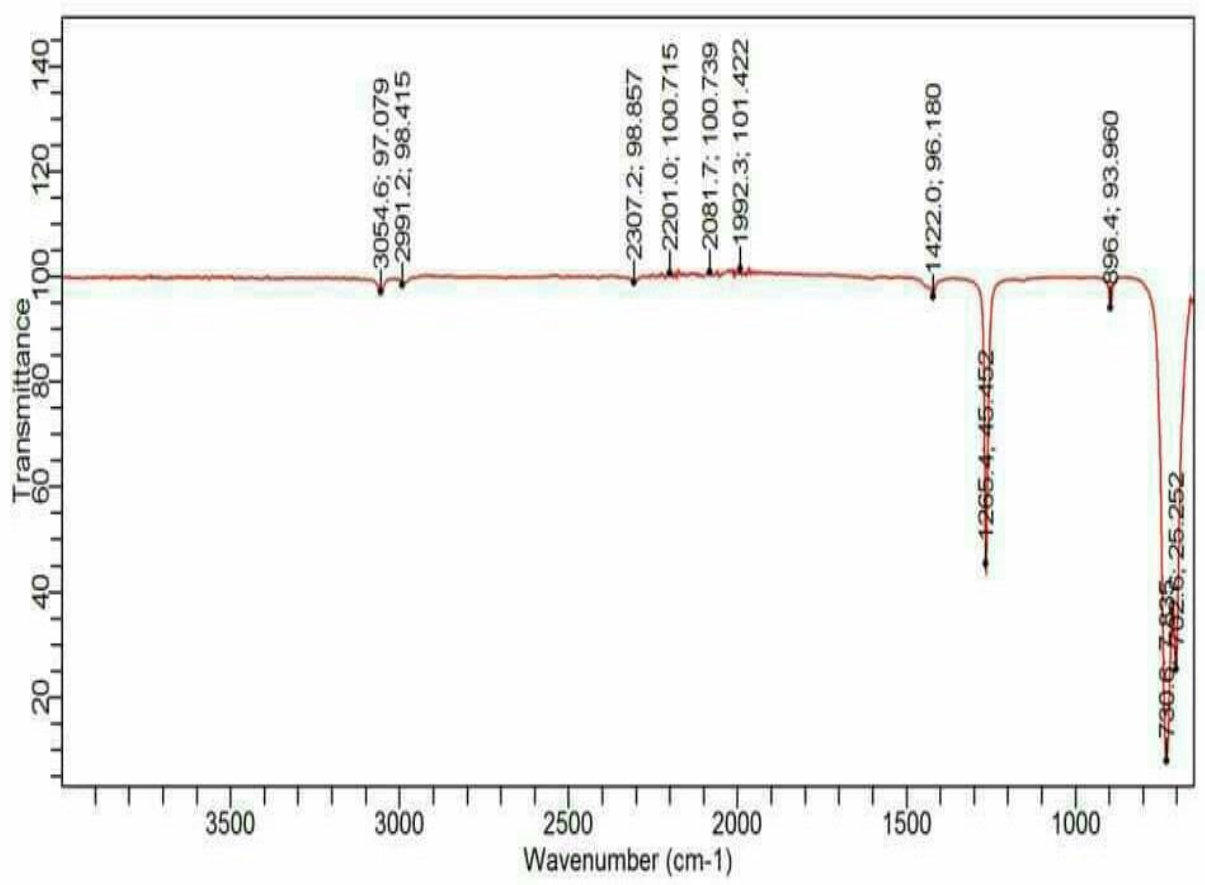

Fig 2.0. The chromatogram of the FTIR analyses of the ethanolic root extract of Datura metel 
African Journal of Biology and Medical Research

ISSN: $2689-534 \mathrm{X}$

Volume 5, Issue 1, 2022 (pp. 30-52)

www.abjournals.org

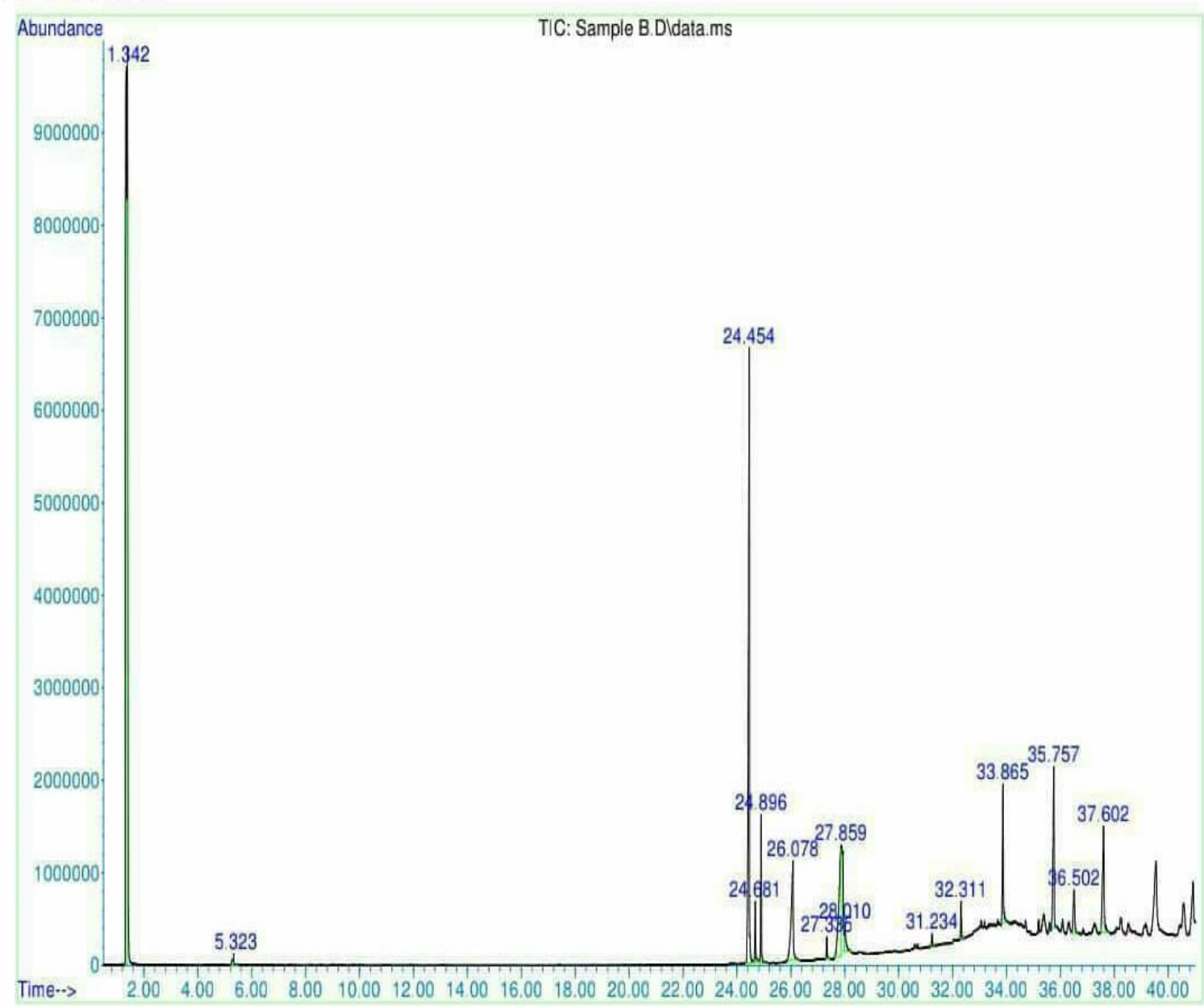

Fig 3.0. The chromatogram of the GC-MS analyses of the ethanolic Root extract of Datura metel 
African Journal of Biology and Medical Research

ISSN: $2689-534 \mathrm{X}$

Volume 5, Issue 1, 2022 (pp. 30-52)

www.abjournals.org

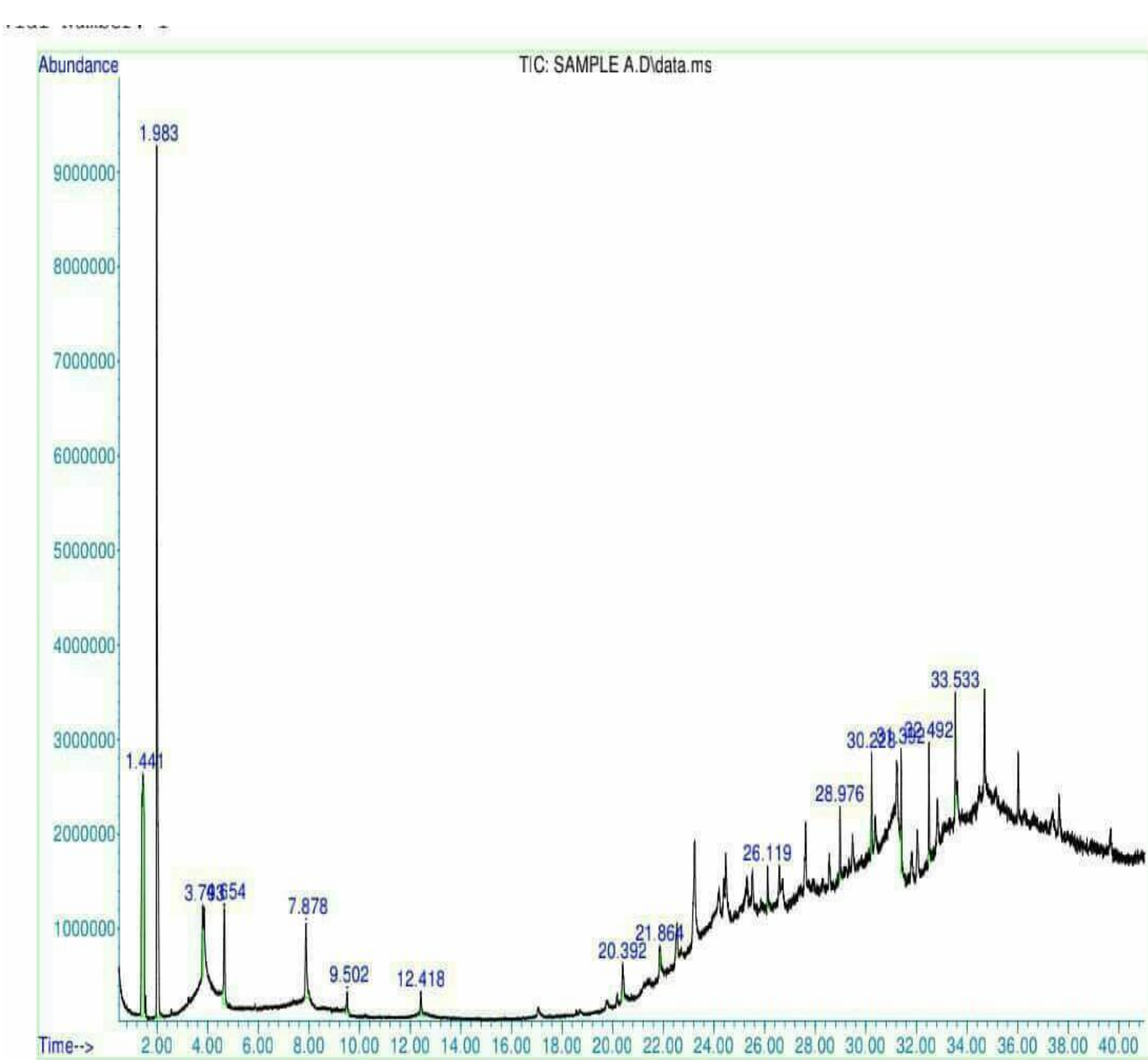

Fig 4.0. The chromatogram of the GC-MS analyses of the ethanolic leaf extract of Datura metel 
African Journal of Biology and Medical Research

ISSN: $2689-534 \mathrm{X}$

Volume 5, Issue 1, 2022 (pp. 30-52)

www.abjournals.org

Table 3.1. Results on the preliminary phytochemical screening of ethanolic leaf and root extract of Datura metel.

\begin{tabular}{|lc|}
\hline PHYTOCHEMICAL RESULTS/INFERENCE & \\
\hline ALKALOID & + \\
SAPONINS & + \\
TANINS & + \\
FLAVONOIDS & + \\
TERPENES & + \\
\hline
\end{tabular}

KEY + = PRESENT

Table 3.2. Results for FT-IR analysis of ethanolic Leaf extract of Datura metel

\begin{tabular}{|ccc|}
\hline S/N & Wavelenght & Functional Group \\
\hline 1 & 3054.6 & $=\mathrm{C}-\mathrm{H}$ \\
2 & 2987.6 & $\mathrm{C}-\mathrm{H}$ \\
3 & 2920 & $\mathrm{C}-\mathrm{H}$ \\
4 & 2309.1 & Isothiocyanate (-NCS) \\
5 & 2152.5 & Isothiocyanate (-NCS) \\
6 & 2005.3 & Isothiocyanate (-NCS) \\
7 & 1932.6 & Amines \\
8 & 1442 & Methyl (C-H) \\
9 & 1265.4 & C-N \\
10 & 896.4 & C-H \\
11 & 730 & $-(\mathrm{CH})_{\mathrm{n}}$ \\
12 & 702.6 & $\mathrm{C}-\mathrm{Cl}$ \\
& & \\
\hline
\end{tabular}

Table 3.3.Results for FT-IR analysis of ethanolicRoot extract of Datura metel

\begin{tabular}{|ccc|}
\hline S/N & Wavelenght & Functional Group \\
\hline 1 & 3054.6 & $=\mathrm{C}-\mathrm{H}$ \\
2 & 2997.2 & $-\mathrm{C}-\mathrm{H}$ \\
3 & 2201 & $-\mathrm{C}=\mathrm{C}-$ \\
4 & 2081.7 & Transition metal carbonyls \\
5 & 1992.3 & Transition metal carbonyls \\
6 & 1422 & $\mathrm{C}=\mathrm{C}$ \\
7 & 1265.4 & $\mathrm{C}-\mathrm{O}$ \\
8 & 896.4 & $\mathrm{C}-\mathrm{H}$ \\
9 & 730.6 & $\mathrm{C}-\mathrm{Cl}$ \\
10 & 702.6 & $-\mathrm{OH}$ \\
\hline
\end{tabular}


African Journal of Biology and Medical Research

ISSN: 2689-534X

Volume 5, Issue 1, 2022 (pp. 30-52)

www.abjournals.org

Table 3.4 Result for GC-MS Analysis of the ethanolic Leaf extract of Datura metel

\begin{tabular}{|c|c|c|c|c|c|}
\hline $\begin{array}{l}\mathrm{S} / \\
\mathrm{N} \\
\mathrm{O}\end{array}$ & $\begin{array}{l}\text { Retention } \\
\text { time(R.T) } \\
\text { (Mins) }\end{array}$ & Name of compound & $\begin{array}{l}\text { Molecular } \\
\text { weight }\end{array}$ & $\begin{array}{l}\text { Molecular } \\
\text { formular }\end{array}$ & $\begin{array}{l}\% \\
\text { composi } \\
\text { tion }\end{array}$ \\
\hline 1 & 1.441 & $\begin{array}{l}\text { Adenosine,4'de (hydroxymethyl)-4'- } \\
\text { (N-ethylaminoformyl }\end{array}$ & 442 & $\begin{array}{l}\mathrm{C}_{20} \mathrm{H}_{22} \mathrm{~N}_{6} \mathrm{O} \\
6\end{array}$ & 20.804 \\
\hline 2 & 1.441 & Chlorozotocin & 313 & $\mathrm{C}_{9} \mathrm{H}_{1 \sigma} \mathrm{ClN}_{3} \mathrm{O}$ & 20.804 \\
\hline 3 & 1.983 & 2-methyl-3-thiosemicarbazide & 105 & $\mathrm{C}_{2} \mathrm{H}_{7} \mathrm{~N}_{3} \mathrm{~S}$ & 38.621 \\
\hline 4 & 1.983 & $\begin{array}{l}\text { Benzenehexanenitrile }, \beta, \beta \text {-dimethyl- } \varepsilon^{-} \\
\text {oxo }\end{array}$ & 215 & $\mathrm{C}_{14} \mathrm{H}_{17} \mathrm{NO}$ & 38.621 \\
\hline 5 & 3.793 & Hexestrol,pentafluoropropionyl- & 416 & $\mathrm{C}_{21} \mathrm{H}_{21} \mathrm{~F}_{5} \mathrm{O}_{3}$ & 3.065 \\
\hline 6 & 3.793 & $\begin{array}{l}\text { (1,2-Diethylethylene)bis(phenylene) } \\
\text { diacetate }\end{array}$ & 354 & $\mathrm{C}_{22} \mathrm{H}_{26} \mathrm{O}_{4}$ & 3.065 \\
\hline 7 & 4.654 & $\begin{array}{l}\text { 3- } \\
\text { Methylbenzylalcohol,trifluoroacetate }\end{array}$ & 218 & $\mathrm{C}_{10} \mathrm{H}_{9} \mathrm{~F}_{3} \mathrm{O}_{2}$ & 5.575 \\
\hline 8 & 4.654 & $\begin{array}{l}\text { 4-Methylbenzyl alcohol, } \\
\text { trifluoroacetate }\end{array}$ & 218 & $\mathrm{C}_{10} \mathrm{H}_{9} \mathrm{~F}_{3} \mathrm{O}_{2}$ & 5.575 \\
\hline 9 & 7.878 & $\begin{array}{l}\text { Trisiloxane }, 1,1,1,5,5,5 \text {-hexamethyl- } \\
\text { 3-〔(trimethylsily)oxy } 〕\end{array}$ & 296 & $\begin{array}{l}\mathrm{C}_{9} \mathrm{H}_{28} \mathrm{O}_{3} \mathrm{Si} \\
4\end{array}$ & 5.282 \\
\hline 10 & 7.878 & Cyclotetrasiloxane,octamethyl- & 296 & $\mathrm{C}_{8} \mathrm{H}_{24} \mathrm{O}_{4} \mathrm{Si}_{4}$ & 5.282 \\
\hline 11 & 9.502 & Estragole & 148 & $\mathrm{C}_{10} \mathrm{H}_{12} \mathrm{O}$ & 1.521 \\
\hline 12 & 9.502 & Anethole & 148 & $\mathrm{C}_{10} \mathrm{H}_{12} \mathrm{O}$ & 1.521 \\
\hline 13 & 12.418 & (-)-Epigallocatechin & 290 & $\mathrm{C}_{15} \mathrm{H}_{14} \mathrm{O}_{6}$ & 1.580 \\
\hline 14 & 12.418 & Catechin & 290 & $\mathrm{C}_{15} \mathrm{H}_{14} \mathrm{O}_{6}$ & 1.580 \\
\hline 15 & 20.392 & Ellagic acid & 302 & $\mathrm{C}_{14} \mathrm{H}_{6} \mathrm{O}_{8}$ & 2.632 \\
\hline 16 & 20.392 & $\begin{array}{l}\text { 1,1'-Biphenyl] -3,3'- } \\
\text { dicarboxaldehyde,6,6'-dihydroxy- } \\
\text { 5,5'-dimethoxyl }\end{array}$ & 302 & $\mathrm{C}_{16} \mathrm{H}_{14} \mathrm{O}_{6}$ & 2.632 \\
\hline 17 & 21.864 & Vitamin E & 430 & $\mathrm{C}_{29} \mathrm{H}_{50} \mathrm{O}_{2}$ & 0.797 \\
\hline 18 & 21.864 & $(+)_{-\gamma}$-Tocopherol,o-methyl- & 430 & $\mathrm{C}_{29} \mathrm{H}_{50} \mathrm{O}_{2}$ & 0.797 \\
\hline 19 & 26.119 & $\begin{array}{l}\text { 3-Pyridinecarboxylicacid,2,7,10- } \\
\text { tris(acetyloxy)- } \\
\text { 1,1a,2,3,4,6,7,10,11,11a-decahydro- } \\
\text { 1,1,3,6,9-pentamethyl-4-oxo-4a,7a- } \\
\text { epoxy-5H-cyclopenta〔a〕 } \\
\text { cyclopropa〔f〕 cycloundecen-11-yl } \\
\text { ester,〔1Ar- } \\
1 \mathrm{aR} *, 2 \mathrm{R}^{*}, 3 \mathrm{~S}^{*}, 4 \mathrm{aR} *, 6 \mathrm{~S}^{*}, 7 \mathrm{~S}^{*}, 7 \mathrm{aS} *, 8 \\
\left.\mathrm{E}, 10 \mathrm{R}^{*}, 11 \mathrm{R}^{*}, 11 \mathrm{As} \mathrm{s}^{*}\right)\end{array}$ & 597 & $\mathrm{C}_{32} \mathrm{H}_{39} \mathrm{NO}_{1}$ & 1.551 \\
\hline
\end{tabular}


African Journal of Biology and Medical Research ISSN: 2689-534X

Volume 5, Issue 1, 2022 (pp. 30-52)

www.abjournals.org

\begin{tabular}{|c|c|c|c|c|c|}
\hline 20 & 26.119 & $\begin{array}{l}\text { Hexadecanoic 1a,2,5,5a,6,9,10,10a- } \\
\text { octahydro-5,5a-dihydroxy-4- } \\
\text { (hydroxymethyl)-1,1,7,9- } \\
\text { tetramethyl-11-oxo-1H-2,8a- } \\
\text { methanocyclopenta〔a〕cyclopropa } \\
\text { 〔e〕cyclodecen-6-yl ester, 〔1aR- } \\
(1 \mathrm{a}, 9 \mathrm{a}, 10 \mathrm{a}) 〕\end{array}$ & 586 & $\mathrm{C}_{36} \mathrm{H}_{58} \mathrm{O}_{6}$ & 1.551 \\
\hline 21 & 28.976 & Lorazepam & 320 & $\begin{array}{l}\mathrm{C}_{15} \mathrm{H}_{10} \mathrm{Cl}_{2} \\
\mathrm{~N}_{2} \mathrm{O}_{2}\end{array}$ & 2.630 \\
\hline 22 & 28.976 & $\begin{array}{l}\text { Quinazoline-2-carboxaldehyde,6- } \\
\text { chloro-4-(2-chlorophenyl)- }\end{array}$ & 320 & $\begin{array}{l}\mathrm{C}_{15} \mathrm{H}_{8} \mathrm{Cl}_{2} \\
\mathrm{~N}_{2}\end{array}$ & 2.630 \\
\hline 23 & 30.228 & $\begin{array}{l}\text { 2,4-Imidazolidinedione,5-[3,4- } \\
\text { bis[(trimethylsily)oxy]phenyl]-3- } \\
\text { methyl-5-phenyl-1-(trimethylsilyl }\end{array}$ & 516 & $\begin{array}{c}\mathrm{C}_{25} \mathrm{H}_{40} \mathrm{~N}_{2} \mathrm{O} \\
{ }_{4} \mathrm{Si}_{3}\end{array}$ & 3.006 \\
\hline 24 & 30.228 & $\begin{array}{l}\text { (+)-Prostaglandin } \mathrm{F}^{\delta}, 4 \mathrm{TMS} \\
\text { Derivatives }\end{array}$ & 642 & $\begin{array}{l}\mathrm{C}_{32} \mathrm{H}_{66} \mathrm{O}_{5} \mathrm{~S} \\
\mathrm{i}_{4}\end{array}$ & 3.006 \\
\hline 25 & 31.392 & Benzofuran-2-Carboxylic acid & 162 & $\mathrm{C}_{9} \mathrm{H}_{6} \mathrm{O}_{3}$ & 4.396 \\
\hline 26 & 31.392 & Benzofuran-5-carboxylic acid & 162 & $\mathrm{C}_{9} \mathrm{H}_{6} \mathrm{O}_{3}$ & 4.396 \\
\hline 27 & 32.492 & PGF2 $\breve{\alpha}$ & 354 & $\mathrm{C}_{20} \mathrm{H}_{34} \mathrm{O}_{5}$ & 4.485 \\
\hline 28 & 32.492 & $\begin{array}{l}\text { Aceticacid,1-acetoxyl-10a,12a- } \\
\text { dimethyl-5-oxo-hexadecahydro-6- } \\
\text { oxabenzo[3,4]ctclohepta[1,2- } \\
\text { E]inden-8-yl ester }\end{array}$ & 406 & $\mathrm{C}_{23} \mathrm{H}_{34} \mathrm{O}_{6}$ & 4.485 \\
\hline 29 & 33.533 & $\breve{\alpha}$-Tocopheryl acetate & 472 & $\mathrm{C}_{31} \mathrm{H}_{52} \mathrm{O}_{3}$ & 4.054 \\
\hline 30 & 33.533 & $( \pm)-\breve{\alpha}$-Tocopherol acetate & 472 & $\mathrm{C}_{31} \mathrm{H}_{52} \mathrm{O}_{3}$ & 4.054 \\
\hline
\end{tabular}


African Journal of Biology and Medical Research

ISSN: 2689-534X

Volume 5, Issue 1, 2022 (pp. 30-52)

www.abjournals.org

Table 3.5 Result for GC-MS Analysis of the ethanolic root extract of Datura metel

\begin{tabular}{|c|c|c|c|c|c|}
\hline $\mathrm{S} / \mathrm{N}$ & $\begin{array}{l}\text { Retention } \\
\text { time } \\
(\mathrm{R} . \mathrm{T})(\mathrm{Mins}) \\
\end{array}$ & Name of compound & $\begin{array}{l}\text { Molecular } \\
\text { weight }\end{array}$ & $\begin{array}{l}\text { Molecular } \\
\text { formular }\end{array}$ & $\begin{array}{l}\% \\
\text { compo } \\
\text { sition }\end{array}$ \\
\hline 1 & 1.342 & Thiophene,2,3-dihydro & 86 & $\mathrm{C}_{4} \mathrm{H}_{6} \mathrm{~S}$ & 38.914 \\
\hline 2 & 1.342 & Thiophene,2,5 - dihydro & 86 & $\mathrm{C}_{4} \mathrm{H}_{6}$ & 38.914 \\
\hline 3 & 5.323 & Bis(2 - ethylhexyl)phytalate & 390 & $\mathrm{C}_{24} \mathrm{H}_{38} \mathrm{O}_{4}$ & 0.542 \\
\hline 4 & 5.323 & Diisooctyl phythalate & 390 & $\mathrm{C}_{24} \mathrm{H}_{38} \mathrm{O}_{4}$ & 0,542 \\
\hline 5 & 24.45 & $\begin{array}{r}\text { Iron }(1,2-\text { ethanediyl/bis }(1-\eta \\
- \text { ethyl }-2,4-\text { cy }\end{array}$ & 426 & $\mathrm{C}_{22} \mathrm{H}_{44} \mathrm{FeP}_{2}$ & 19.744 \\
\hline 6 & 24.45 & $\begin{array}{r}\text { Hydrido-iron, }[n-5(1-\text { ethlcuc } \\
- \text { bis }(\text { diisopropylph }\end{array}$ & 426 & $\mathrm{C}_{22} \mathrm{H}_{44} \mathrm{FeP}$ & 19.744 \\
\hline 7 & 24.68 & $\begin{array}{l}3,7,11,15 \text {-Tetramethyl-2- } \\
\text { hexadecen-1 } \\
\text {-ol }\end{array}$ & 296 & $\mathrm{C}_{20} \mathrm{H}_{40} \mathrm{O}$ & 1.497 \\
\hline 8 & 24.68 & Phytolacetate & 338 & $\mathrm{C}_{20} \mathrm{H}_{42} \mathrm{O}_{2}$ & 1.497 \\
\hline 9 & 24.89 & Phytylhexadecanoate & 534 & $\mathrm{C}_{36} \mathrm{H}_{70} \mathrm{O}_{2}$ & 3.664 \\
\hline 10 & 24.89 & $\begin{array}{l}\text { hexadecanoicacid,3,7,11,15- } \\
\text { tetramethyl-2-hexadecenylester, } \\
\text { 〔R-〔R,R-E〕 }\end{array}$ & 534 & $\mathrm{C}_{36} \mathrm{H}_{70} \mathrm{O}_{2}$ & 3.664 \\
\hline 11 & 26.07 & $\begin{array}{l}\text { I-(+)-Ascorbicacid 2,6- } \\
\text { dihexadecanote }\end{array}$ & 652 & $\mathrm{C}_{38} \mathrm{H}_{68} \mathrm{O}_{8}$ & 6.501 \\
\hline 12 & 26.07 & n-Hexadecanoic acid & 256 & $\mathrm{C}_{16} \mathrm{H}_{32} \mathrm{O}_{2}$ & 6.501 \\
\hline 13 & 27.335 & Ethyl iso-alocholate & 436 & $\mathrm{C}_{26} \mathrm{H}_{44} \mathrm{O}_{5}$ & 0.568 \\
\hline 14 & 27.335 & 1-Heptatriacotanol & 536 & $\mathrm{C}_{37} \mathrm{H}_{76} \mathrm{O}$ & 0.568 \\
\hline 15 & 27.85 & Linoelaidic acid & 280 & $\mathrm{C}_{18} \mathrm{H}_{32} \mathrm{O}_{2}$ & 8.403 \\
\hline 16 & 27.85 & (Z)-18-Octadec-9-enolide & 280 & $\mathrm{C}_{18} \mathrm{H}_{32} \mathrm{O}_{2}$ & 8.403 \\
\hline 17 & 28.010 & $\begin{array}{l}\text { 4-Androstene-3,17-dione } 17 \text { - } \\
\text { mono(O-methyloxime }\end{array}$ & 315 & $\mathrm{C}_{20} \mathrm{H}_{29} \mathrm{NO}_{2}$ & 1.188 \\
\hline 18 & 28.010 & $\begin{array}{l}\text { 5,19-cyclo-5ß-androst-6-ene-3,17- } \\
\text { dione }\end{array}$ & 284 & $\mathrm{C}_{20} \mathrm{H}_{29} \mathrm{NO}_{2}$ & 1.188 \\
\hline
\end{tabular}


African Journal of Biology and Medical Research

ISSN: 2689-534X

Volume 5, Issue 1, 2022 (pp. 30-52)

www.abjournals.org

\begin{tabular}{|c|c|c|c|c|c|}
\hline 19 & 31.234 & $\begin{array}{l}\text { 3',8,8'-Teimethoxy-3-piperidyl- } \\
\text { 2,2'-binaphthalene-1,1',4,4'- } \\
\text { tetrone }\end{array}$ & 487 & $\mathrm{C}_{28} \mathrm{H}_{25} \mathrm{NO}_{7}$ & 0.277 \\
\hline 20 & 31.234 & $\begin{array}{l}\text { 6,19-Cycloandrostane-3,7- } \\
\text { diol,3ß- } \\
\text { methoxyl }\end{array}$ & 320 & $\mathrm{C}_{20} \mathrm{H}_{32} \mathrm{O}_{3}$ & 0.277 \\
\hline 21 & 32.311 & (S)-(+)-Epichlorohydrin & 92 & $\mathrm{C}_{3} \mathrm{H}_{5} \mathrm{CIO}$ & 0.859 \\
\hline 22 & 32.311 & Oxirane(chloromethyl) & 92 & $\mathrm{C}_{3} \mathrm{H}_{5} \mathrm{CIO}$ & 0.859 \\
\hline 23 & 33.865 & 9,10-Anthracenedione & 208 & $\mathrm{C}_{14} \mathrm{H}_{8} \mathrm{O}_{2}$ & 4.339 \\
\hline 24 & 33.865 & 9,10-Phenanthrenedione & 208 & $\mathrm{C}_{14} \mathrm{H}_{8} \mathrm{O}_{2}$ & 4.339 \\
\hline 25 & 35.757 & Amodiaquine & 355 & $\begin{array}{l}\mathrm{C}_{20} \mathrm{H}_{22} \mathrm{CL} \\
\mathrm{N}_{3} \mathrm{O}\end{array}$ & 6.118 \\
\hline 26 & 35.757 & Amopyroquine & 355 & $\begin{array}{l}\mathrm{C}_{20} \mathrm{H}_{22} \mathrm{CL} \\
\mathrm{N}_{3} \mathrm{O}\end{array}$ & 6.118 \\
\hline 27 & 36.502 & ß-Tocopherol,o-methl & 430 & $\mathrm{C}_{29} \mathrm{H}_{50} \mathrm{O}_{2}$ & 2.003 \\
\hline 28 & 36.502 & $(+)_{\gamma}-$ Tocopherol,o-methyl & 430 & $\mathrm{C}_{29} \mathrm{H}_{50} \mathrm{O}_{2}$ & 2.003 \\
\hline 29 & 37.602 & $\begin{array}{l}\text { 7H-Pyranol〔2,3-c } \text { acridin-7- } \\
\text { one, } \\
\text { 3,12-dihydro-6,11-dihydroxy- } \\
\text { 3,3,12- } \\
\text { Trimethyl-5-(3-methyl-2-butenyl) }\end{array}$ & 391 & $\mathrm{C}_{24} \mathrm{H}_{25} \mathrm{NO}_{4}$ & 5.382 \\
\hline 30 & 37.602 & $\begin{array}{l}\text { 1-Azaspiro〔4.5〕 dec-3-ene,2- } \\
\text { (diphenylmethylene)-4-methyl-1- } \\
\text { phenyl }\end{array}$ & 391 & $\mathrm{C}_{29} \mathrm{H}_{29} \mathrm{~N}$ & 5.382 \\
\hline
\end{tabular}

\section{DISCUSSION}

The phytochemical Evaluation of leaf and root ethanolic extract of Datura metel showed presence of alkaloid, saponins, tannins, flavonoid and terpenes. Studies are in line with results from Datura metel Linn by sundaramoorthy, 2014. Phytochemical are known for their biological activities such as antimicrobial, antioxidant, antifungal, anticancer and antidiabetic strength (Hossain, 2011). Tannins, Saponins, Flavonoids have anti-hypoglycaemic and antiinflammatory properties, terpenes have analgesic properties and as well as central nervous system (CNS) activity (Ayoola etal, 2008). Alkaloids have antioxidant, antifungal, protective properties. 
Fourier Transform Infrared Spectroscopy (FTIR) of both leaf and root ethanolic extracts of plant, showed presence of functional groups such as alkanes, alkenes, amines, isothicyanates and alkyl halides The wavelength study was in line with observation from Cleome gynandra leaf (Deepashree etal, 2013) were the band range between 20005.3m-2997.2m were transition metals and isothiocynate (-NCS). Wavelength of $2987 \mathrm{~m}$ and $2920 \mathrm{~m}$ indicates presence of compounds such as methyl $(-\mathrm{C}-\mathrm{H})$. Wavelength of $3054 \mathrm{~m}$ showed presence of alkene $(\mathrm{C}=\mathrm{C})$. Wavelength of $1990 \mathrm{~m}-2150 \mathrm{~m}$ showed presence of nitrogen multiple and cumulative double bound compounds isothiocyanate (-NCS). Wavelength of $1932 \mathrm{~m}$ indicates primary amines. Wavelength of $1150 \mathrm{~m}-1210 \mathrm{~m}$, aliphatic chloro compound (C-CL). Wavelength within $1200 \mathrm{~m}-$ $1800 \mathrm{~m}$, showed transition metals and Wavelength of 590m-720m indicated Alcohol (-OH) and Hydroxyl compounds. (Coates 2000).

The results from GC-MS analysis showed that roots and leaves extracts of datura metel contained so many bioactive compounds belonging to various classes of phytochemicals which plays some biological roles in the body system as discussed in tables 4.1 and 4.2.

Table 4.1 Biological Activity of Identified Compound in Ethanolic Leaf extract of Datura metel.

\begin{tabular}{|c|c|c|c|}
\hline S/No & Compound Biological Activity & Uses & Reference \\
\hline 1 & $\begin{array}{l}\text { Adenosine, } 4 \text { ' de(hydroxymethyl })-4 \text { '-(N- } \\
\text { ethylaminoformyl) }\end{array}$ & $\begin{array}{l}\text { Binds readily with } \\
\text { adenosine than to } \\
\text { other endogenous } \\
\text { purines with two } \\
\text { pharmacologically } \\
\text { distinguishable type } \\
\left(a_{1} \text { and }_{2}\right) \text { to exert } \\
\text { biological effect. }\end{array}$ & NCBI, 2021. \\
\hline 2 & Chlorozotocin & $\begin{array}{l}\text { A nitroso urea used } \\
\text { for cancer therapy } \\
\text { and analogue of } \\
\text { streptozotocin }\end{array}$ & Cooke, 2006. \\
\hline 3 & $2-$ methyl-3-thiosemicarbazide & $\begin{array}{l}\text { Exhibit an anti- } \\
\text { arrangement between } \\
\text { thione } \mathrm{S} \text { atom and } \\
\text { hydrazine } \mathrm{N} \text { atom }\end{array}$ & $\begin{array}{l}\text { Jesus Valdes- } \\
\text { Martinez, } 2007 .\end{array}$ \\
\hline 4 & Benzenehexanenitrile, ${ }_{\beta}, \beta-$ dimethyl $-\varepsilon-$ oxo & $\begin{array}{l}\text { Is an Aromatic } \\
\text { ketone }\end{array}$ & TSCA,2006 \\
\hline 5 & Hexestrol, pentafluoropropionyl- & $\begin{array}{l}\text { Non-steriodal } \\
\text { estrogen previously } \\
\text { used for estrogen- } \\
\text { replacement therapy } \\
\text { and treatment of } \\
\text { certain hormone } \\
\text { dependent cancers. }\end{array}$ & J.elks (2014). \\
\hline 6 & $(1,2-$ Diethylethylene $)$ bis(phenylene) diacetate & No activity reported & Nil \\
\hline 7 & 3-Methylbenzylalcohol,trifluoroacetate & No activity reported & Nil \\
\hline
\end{tabular}


African Journal of Biology and Medical Research

ISSN: 2689-534X

Volume 5, Issue 1, 2022 (pp. 30-52)

www.abjournals.org

\begin{tabular}{|c|c|c|c|}
\hline 8 & 4-Methylbenzyl alchol, trifluoroacetate & No activity reported & Nil \\
\hline 9 & $\begin{array}{l}\text { Trisiloxane }, 1,1,1,5,5,5-\text { hexamethyl }-3- \\
\text { [(trimethylsily)oxy }\end{array}$ & No activity reported & Nil \\
\hline 10 & Cyclotetrasiloxane, octamethyl- & $\begin{array}{l}\text { Used in manufacture } \\
\text { of polymeric } \\
\text { materials widely used } \\
\text { in cosmetics }\end{array}$ & EPA.2016 \\
\hline 11 & Estragole & $\begin{array}{l}\text { Used as flavouring } \\
\text { agent in } \\
\text { pharmaceutical } \\
\text { industry, cosmetic } \\
\text { and food industry, } \\
\text { antioxidant, } \\
\text { antimicrobial } \\
\text { properties. }\end{array}$ & Friedman,2002 \\
\hline 12 & Anethole & $\begin{array}{l}\text { Is the main fragrance } \\
\text { and bioactive } \\
\text { compound in some } \\
\text { plant species. It has } \\
\text { antimicrobial, } \\
\text { antifungal, } \\
\text { antihelmetic and } \\
\text { insecticidal activity }\end{array}$ & Astani,2011 \\
\hline 13 & (-)-Epigallocatechin & $\begin{array}{l}\text { It reduces } \\
\text { inflammation, aid } \\
\text { weight loss, helps } \\
\text { prevent heat and } \\
\text { brain disease. }\end{array}$ & Ansley2019 \\
\hline 14 & Catechin & $\begin{array}{l}\text { It is a phenolic } \\
\text { compound, mostly } \\
\text { found in tea, cocoa } \\
\text { and berries and have } \\
\text { antioxidant actitvity. }\end{array}$ & Tania,2006 \\
\hline 15 & Ellagic acid & $\begin{array}{l}\text { Natural phenol, } \\
\text { antioxidant found in } \\
\text { fruits and vegetables }\end{array}$ & Ryszand,2012 \\
\hline 16 & $\begin{array}{l}\text { 1,1'-Biphenyl] -3,3'-dicarboxaldehyde,6,6'- } \\
\text { dihydroxy-5,5'-dimethoxyl }\end{array}$ & No activity reported & Nil \\
\hline 17 & Vitamin E & $\begin{array}{l}\text { Antiageing, } \\
\text { Analgesic, } \\
\text { Antidiabatic, } \\
\text { Antiinflammatory, } \\
\text { Antioxidant, }\end{array}$ & $\begin{array}{l}\text { Juliana Kubala, } \\
2021 .\end{array}$ \\
\hline 18 & $(+)_{-\gamma}$-Tocopherol,o-methyl- & $\begin{array}{l}\text { Fat soluble } \\
\text { antioxidant }\end{array}$ & $\begin{array}{l}\text { Gamze Guelu, } \\
2021 .\end{array}$ \\
\hline
\end{tabular}


African Journal of Biology and Medical Research

ISSN: 2689-534X

Volume 5, Issue 1, 2022 (pp. 30-52)

www.abjournals.org

\begin{tabular}{|c|c|c|c|}
\hline 19 & $\begin{array}{l}\text { 3-Pyridinecarboxylicacid,2,7,10-tris(acetyloxy)- } \\
\text { 1,1a,2,3,4,6,7,10,11,11a-decahydro-1,1,3,6,9- } \\
\text { pentamethyl-4-oxo-4a,7a-epoxy-5H-cyclopenta } \\
\text { a] cyclopropa 〈f] cycloundecen-11-yl ester, } \\
1 A r_{-} \\
1 a R^{*}, 2 R^{*}, 3 S^{*}, 4 a R^{*}, 6 S^{*}, 7 S^{*}, 7 a S^{*}, 8 E, 10 R^{*}, 11 R^{*} \\
\left., 11 A s^{*}\right)\end{array}$ & No activity reported & Nil \\
\hline 20 & $\begin{array}{l}\text { Hexadecanoic } 1 \text { a, } 2,5,5 a, 6,9,10,10 a-\text { octahydro- } \\
\text { 5,5a-dihydroxy-4-(hydroxymethyl)-1,1,7,9- } \\
\text { tetramethyl-11-oxo-1H-2,8a-methanocyclopenta } \\
\quad\{a\rfloor \text { cyclopropa }\{e\rfloor \text { cyclodecen-6-yl ester, } \\
1 a R-(1 a, 9 a, 10 a)\rfloor\end{array}$ & No activity reported & Nil \\
\hline 21 & Lorazepam & $\begin{array}{l}\text { Used in healing } \\
\text { anxiety disorder, } \\
\text { active seizure, } \\
\text { alcohol withdrawal, } \\
\text { chemotherapy, } \\
\text { induced nausea and } \\
\text { vomiting } \\
\end{array}$ & $\begin{array}{l}\text { Julie Maves } \\
(2021) \text {. }\end{array}$ \\
\hline 22 & $\begin{array}{l}\text { Quinazoline-2-carboxaldehyde,6-chloro-4-(2- } \\
\text { chlorophenyl)- }\end{array}$ & No activity reported & Nil \\
\hline 23 & $\begin{array}{l}\text { 2,4-Imidazolidinedione,5-[3,4- } \\
\text { bis[(trimethylsily)oxy]phenyl]-3-methyl-5- } \\
\text { phenyl-1-(trimethylsilyl }\end{array}$ & No activity reported & Nil \\
\hline 24 & $(+)$-Prostaglandin F2 ${ }^{\delta}, 4 \mathrm{TMS}$ Derivatives & $\begin{array}{l}\text { Notable for } \\
\text { promotion of uterine } \\
\text { contraction }\end{array}$ & $\begin{array}{l}\text { Emmanuel etal, } \\
2011\end{array}$ \\
\hline 25 & Benzofuran-2-Carboxylic acid & $\begin{array}{l}\text { Anti-inflammatory, } \\
\text { local anaesthetics, } \\
\text { cytotoxicity against } \\
\text { human cancer cell } \\
\text { line. }\end{array}$ & Saku etal, 2010. \\
\hline 26 & Benzofuran-5-carboxylic acid & $\begin{array}{l}\text { Antiviral, antioxidant, } \\
\text { anti-inflammatory, } \\
\text { antioxidant, } \\
\text { Antimicrobial, } \\
\text { antitumor activity } \\
\end{array}$ & $\begin{array}{l}\text { Hayakwa etal, } \\
2004\end{array}$ \\
\hline 27 & PGF2 $\breve{\alpha}$ & $\begin{array}{l}\text { Is a stable } \\
\text { prostaglandin that } \\
\text { stimulates the } \\
\text { contraction of uterine } \\
\text { and bronchial smooth } \\
\text { muscle and tightening } \\
\text { in some blood vessels } \\
\end{array}$ & $\begin{array}{l}\text { Jian Zhang etal, } \\
2010 .\end{array}$ \\
\hline
\end{tabular}


African Journal of Biology and Medical Research

ISSN: $2689-534 \mathrm{X}$

Volume 5, Issue 1, 2022 (pp. 30-52)

www.abjournals.org

\begin{tabular}{|l|l|l|l|}
\hline 28 & $\begin{array}{l}\text { Aceticacid,1-acetoxyl-10a,12a-dimethyl-5-oxo- } \\
\text { hexadecahydro-6-oxabenzo[3,4]ctclohepta[1,2- } \\
\text { E]inden-8-yl ester }\end{array}$ & No activity reported & Nil \\
\hline 29 & $\breve{\alpha}$-Tocopheryl acetate & $\begin{array}{l}\text { Also known as } \\
\text { vitamin E acetate and } \\
\text { safe for use in } \\
\text { cosmetics and } \\
\text { skincare products }\end{array}$ & SAS 2016 \\
\hline 30 & $\begin{array}{l}( \pm)-\breve{\alpha} \text {-Tocopherol acetate } \\
\text { vitamin E acetate and } \\
\text { safe for use in } \\
\text { cosmetics and } \\
\text { skincare products }\end{array}$ & SAS 2016 \\
\hline
\end{tabular}

Table 4.2: Biological Activity of Identified Compound in Ethanolic Root extract of Datura metel.

\begin{tabular}{|l|l|l|l|}
\hline $\begin{array}{l}\text { S/ } \\
\text { no }\end{array}$ & Compounds biological activity & Uses & Reference \\
\hline 1 & Thiophene,2,3 - dihydro & $\begin{array}{l}\text { Antimicrobial, } \\
\text { analgesic, anti- } \\
\text { inflammatory, } \\
\text { antihypertensive and } \\
\text { antitumor }\end{array}$ & $\begin{array}{l}\text { Pillai etal, } \\
2005\end{array}$ \\
\hline 2 & Thiophene,2,5-dihydro & $\begin{array}{l}\text { Inhibitors of corrosion } \\
\text { of metals and } \\
\text { fabrication of light- } \\
\text { emitting diode in } \\
\text { material science }\end{array}$ & $\begin{array}{l}\text { Benabdlellh } \\
\text { etal,2006 }\end{array}$ \\
\hline 3 & Bis(2-ethylhexyl)phytalate & $\begin{array}{l}\text { DEHP is used as } \\
\text { plasticizers in many } \\
\text { products, especially in } \\
\text { medicinal device such } \\
\text { as intravenous bags } \\
\text { and tubing, blood } \\
\text { bags, infusion tubing, } \\
\text { peritoneal dialysis } \\
\text { bags. }\end{array}$ & $\begin{array}{l}\text { Hung P.C } \\
\text { etal, 2008. }\end{array}$ \\
\hline 4 & Diisooctyl phythalate & $\begin{array}{l}\text { DIOP is used in rubber } \\
\text { compounds for } \\
\text { manufacture of } \\
\text { automobile hoses and } \\
\text { parts }\end{array}$ & $\begin{array}{l}\text { Kent } \\
\text { Carlson, } \\
2010\end{array}$ \\
\hline
\end{tabular}


African Journal of Biology and Medical Research

ISSN: 2689-534X

Volume 5, Issue 1, 2022 (pp. 30-52)

www.abjournals.org

\begin{tabular}{|c|c|c|c|}
\hline 5 & $\begin{array}{r}\text { Iron }(1,2-\text { ethanediyl } / \text { bis }(1-\text { methylethyl }) \\
- \text { ethyl }-2,4-\text { cyclohexadien }-\end{array}$ & $\begin{array}{l}\text { No activity reported } \\
\text { yet }\end{array}$ & Nil \\
\hline 6 & $\begin{array}{r}\text { Hydrido - iron, }[n-5(1-\text { ethlcuclohexadienyl }) \\
- \text { bis }(\text { diisopropylphosphino }) \text { ethar }\end{array}$ & $\begin{array}{l}\text { No activity reported } \\
\text { yet }\end{array}$ & Nil \\
\hline 7 & $\begin{array}{l}\text { 3,7,11,15-Tetramethyl-2-hexadecen-1 } \\
\text {-ol }\end{array}$ & $\begin{array}{l}\text { No activity reported } \\
\text { yet }\end{array}$ & Nil \\
\hline 8 & Phytolacetate & $\begin{array}{l}\text { Used as a food } \\
\text { addictive and } \\
\text { flavouring agent }\end{array}$ & EU, 2012. \\
\hline 9 & Phytylhexadecanoate & $\begin{array}{l}\text { No activity reported } \\
\text { yet }\end{array}$ & Nil \\
\hline 10 & $\begin{array}{l}\text { hexadecanoicacid,3,7,11,15-tetramethyl-2- } \\
\text { hexadecenylester, 〔R-〔R,R-E } 〕\end{array}$ & $\begin{array}{l}\text { No activity reported } \\
\text { yet }\end{array}$ & Nil \\
\hline 11 & I-(+)-Ascorbicacid 2,6-dihexadecanote & $\begin{array}{l}\text { It is a vitamin C } \\
\text { compound, used for } \\
\text { treating cold, gum } \\
\text { disease acne and } \\
\text { infections. It is also an } \\
\text { antioxidant in the skin } \\
\text { by scavenging and } \\
\text { quenching free } \\
\text { radicals generated by } \\
\text { ultraviolent radiation }\end{array}$ & $\begin{array}{l}\text { Okenwa, } \\
2014\end{array}$ \\
\hline 12 & n-Hexadecanoic acid & $\begin{array}{l}\text { Also called palmitic } \\
\text { acid, has anti- } \\
\text { inflammatory property } \\
\text { and can be seen in } \\
\text { food addictives, or as } \\
\text { surfactants in } \\
\text { cosmetics }\end{array}$ & $\begin{array}{l}\text { Vasudevan., } \\
2012 .\end{array}$ \\
\hline 13 & Ethyl iso-alocholate & $\begin{array}{l}\text { Also called Ethyl } \\
\text { Cholate, is more stable } \\
\text { than other ligands, and } \\
\text { serve as inhibition for } \\
\text { dehydropteroate } \\
\text { synthase, and can also } \\
\text { be used as } \\
\text { antimicrobial agent }\end{array}$ & $\begin{array}{l}\text { Malathi } \\
\text { etal,2016 }\end{array}$ \\
\hline 14 & 1-Heptatriacotanol & $\begin{array}{l}\text { Has anti-cholesterol } \\
\text { effect }\end{array}$ & $\begin{array}{l}\text { Love } \\
\text { junwei, } 2018\end{array}$ \\
\hline 15 & Linoelaidic acid & $\begin{array}{l}\text { It can modulate } \\
\text { cancer, } \\
\text { atherosclerosis, } \\
\text { obesity, tumour and } \\
\text { diabetics }\end{array}$ & $\begin{array}{l}\text { David A, Z. } \\
2013\end{array}$ \\
\hline
\end{tabular}


African Journal of Biology and Medical Research

ISSN: 2689-534X

Volume 5, Issue 1, 2022 (pp. 30-52)

www.abjournals.org

\begin{tabular}{|c|c|c|c|}
\hline 16 & (Z)-18-Octadec-9-enolide & $\begin{array}{l}\text { Has antibacterial, anti- } \\
\text { parasitic properties }\end{array}$ & $\begin{array}{l}\text { Lalthanpuii } \\
\text { etal, } 2019\end{array}$ \\
\hline 17 & $\begin{array}{l}\text { 4-Androstene-3,17-dione17- } \\
\text { mono(O-methyloxime })\end{array}$ & $\begin{array}{l}\text { Is a drug or nutritional } \\
\text { supplement, which } \\
\text { increases testosterone- } \\
\text { estrogen ratio. }\end{array}$ & $\begin{array}{l}\text { Van thuyne } \\
\text { etal, } 2005 .\end{array}$ \\
\hline 18 & $\begin{array}{l}\text { 5,19-cyclo-5ß-androst-6-ene-3,17- } \\
\text { dione }\end{array}$ & $\begin{array}{l}\text { Has androgenic } \\
\text { activity }\end{array}$ & $\begin{array}{l}\text { Charles. } \\
2012\end{array}$ \\
\hline 19 & $\begin{array}{l}\text { 3',8,8'-Teimethoxy-3-piperidyl- } \\
\text { 2,2'-binaphthalene-1,1',4,4'-tetrone }\end{array}$ & $\begin{array}{l}\text { No activity reported } \\
\text { yet }\end{array}$ & Nil \\
\hline 20 & $\begin{array}{l}\text { 6,19-Cycloandrostane-3,7-diol, 3ß- } \\
\text { methoxyl }\end{array}$ & $\begin{array}{l}\text { No activity reported } \\
\text { yet }\end{array}$ & Nil \\
\hline 21 & (S)-(+)-Epichlorohydrin & $\begin{array}{l}\text { Used to produce } \\
\text { glycerol, plastics, } \\
\text { epoxy glues and resins }\end{array}$ & ECN,2003 \\
\hline 22 & Oxirane(chloromethyl) & $\begin{array}{l}\text { Also known as } \\
\text { ethylene oxide, used to } \\
\text { make detergents, } \\
\text { thickeners, solvents. }\end{array}$ & $\begin{array}{l}\text { Boogaard } \\
\text { P.J.2014 }\end{array}$ \\
\hline 23 & 9,10-Anthracenedione & $\begin{array}{l}\text { Also called } \\
\text { Antraquine, serves as } \\
\text { building blocks for } \\
\text { dyes, bleaches, pulp } \\
\text { for paper making }\end{array}$ & $\begin{array}{l}\text { IUPAC } \\
2014\end{array}$ \\
\hline 24 & 9,10-Phenanthrenedione & $\begin{array}{l}\text { Serves as initial } \\
\text { mediator for electron } \\
\text { acceptor/donor } \\
\text { containing formate } \\
\text { dehydrogenase, } \\
\text { reduction of carbon } \\
\text { dioxide to formate. }\end{array}$ & Robert,2013 \\
\hline 25 & Amodiaquine & $\begin{array}{l}\text { Medication used in } \\
\text { treating malaria, }\end{array}$ & $\begin{array}{l}\text { Nair etal, } \\
2012\end{array}$ \\
\hline 26 & Amopyroquine & $\begin{array}{l}\text { Medication against } \\
\text { chloroquin resisitant } \\
\text { strain of Plasmodium } \\
\text { falciparuim }\end{array}$ & $\begin{array}{l}\text { Lewis } \\
\text { Noble,2012 }\end{array}$ \\
\hline 27 & B-Tocopherol,o-methl & $\begin{array}{l}\text { Has antioxidant } \\
\text { properties }\end{array}$ & $\begin{array}{l}\text { Zing etal, } \\
2013\end{array}$ \\
\hline 28 & $(+){ }_{-\gamma}-$ Tocopherol,o-methyl & $\begin{array}{l}\text { Scavenge ROS during } \\
\text { lipid oxidation }\end{array}$ & $\begin{array}{l}\text { Zing etal, } \\
2013\end{array}$ \\
\hline 29 & $\begin{array}{l}\text { 7H-Pyranol〔2,3-c } 〕 \text { acridin-7-one, } \\
\text { 3,12-dihydro-6,11-dihydroxy-3,3,12- } \\
\text { Trimethyl-5-(3-methyl-2-butenyl) }\end{array}$ & $\begin{array}{l}\text { No activity reported } \\
\text { yet }\end{array}$ & Nil \\
\hline
\end{tabular}




\begin{tabular}{|l|l|l|l|}
\hline 30 & $\begin{array}{l}\text { 1-Azaspiro 〔4.5〕 dec-3-ene,2- } \\
\text { (diphenylmethylene)-4-methyl-1- } \\
\text { phenyl }\end{array}$ & $\begin{array}{l}\text { No activity reported } \\
\text { yet }\end{array}$ & Nil \\
\hline
\end{tabular}

\section{CONCLUSION}

Presence of various bioactive compounds in both plant extracts reveals the biological, pharmacological and industrial strength of the Datura metel. Antioxidants, anticancer, hypocholesterolemic, hypoglycemic, antibacterial activities, as seen in compounds got from phytochemical screening, proves that plant has many medicinal properties. Root extracts effect of plant as seen in epichlorohydrin shows plants importance in industrial use as they can exert harmless or harmful effects. In the industy. Results from this study has justified that datura metel leaf and roots may be very useful in pharmaceutical, health, medicinal and industrial applications for the welfare of human

\section{REFERENCES}

Adegoke S.A. and Alo, L.A. (2013).Datura stromonuim poisioning in children. Nigeria Journal of Clinical Practice .16(1):116-118.

Aiyadurai N., Muthu M., Palaniyandi S. and Rajangam U. (2017). Nematicidal activity of aqueous leaf extracts of Datura metel, Datura innoxia and Brugmansiasuaveolens. American Journal of Entomology 1(2): pp.39-45

Akharaiyi F.C. (2011). Antibacterial and antioxidant activities of Datura metel. International Journal Pharm.Tec.Rex. 3(1):478-483.

Alabiri T.H., MusalamiA. H., Hossia,M.A. ,Weli A.M and AL-Riyami Q. (2014). Comparative study of phytochemical screening, antioxidant and antimicrobial capacities of crude plant of Datura metel 1. Journal King Univ, Sci 26:237-243.

Ali Esmail Al-Snafi. (2017). Medicinal importance of Datura Fatuosa and Datura stramonium-a review. IOSR Journal of Pharmacy.7 (2): 43-58.

Anitha M., Punitha M and Leena G.B. (2014). Phytochemical screening of Datura metel linn and its antimicrobial activity on selected human pathogens. International Journal of Pharmacy.7 (2):43-58.

Ansley Hill (2019). EGCE (Epigallocatechin gallate). Benefits, dosages and safety- a review. Journal of nutrition. 100: 23-27.

Astani A., Reichling J and Scnitzler P. (2011). Screening for antiviral activities of isolated compunds from essential oils. Evidence based complement alternative. Journal of Medicine. 253643:1-8.

Ayoola, G.A., Coker H.A., Adesegun, S.A., Adepoj Bello A.A., Obaweya K., Ezennia and Atangbayilla T.O. (2008). Phytochemical screening and oxidant activities of aqueous and methanol stem extracts of scostusafeker gawl (costaceae). African Journal of Biotechnology 9; 31 4880- 4884

Babalola S.A. (2014). Datura metel Analgesic or Hallucinogen? 'sharo' prospective. Middle East Journal of Scientific Research. 21(6):993-997.

Bauzidi A., Mahdeb. N and Kara .N. (2011). Toxicity studies of alkaloid of seeds of Datura stramonuim and synthesis alkaloids in male rats. Journal Medicinal Plants Research.5 (15): 342-343. 
Benabdellah M., Yahyi A., Dafali A., Hammouli B, Benabdellah M., yahyi A and Etthouhami A (2006). Investigation of inhibitive effect of triphenyltins. 2thiophene carboxylation corrosion of steel in $2 \mathrm{~m}$. HPO solution. Application Surf.Science .252:8341-8347.

Beta S., Dawaung C., Bot C., Abraham K., Abdulateef H., Agwu E., Kujul N and Udokainyang A.D. (2018). Plant remedies used for livestock by farmers in southern senatorial zone, Plateau State. Nigeria. European Journal of Medicinal Plants. 25 (2):1-15

Boogard P.J., Van Puijvelde. M.J and Urbanus J.H (2014). Biological monitoring to accesse dermal exposure to ethylene oxide vapors during air incidental release. Toxicology Letters 231,387-390.

Charles D. Kochakian (2012). Anabolic-Androgenic Steroid. Springer Science and Business Media. Pp171-115

Chavhan S.A, Kadam S.D. and Sapkal P.N. (2018). Pharmacognostic Review on Datura metel. International Journal of Pharmacognosy and Chineese Medicine. 2(4): 600.

Cooke M.M., (2006). Therapy of digestion disorder. Elsevier Health Science. pg 477. ISBN.978-1-4160-01375.

Coates.J. (2000). Interpretation of Infrared Spectra: A potential approach In: Meyers R.A., ED, Encyclopedia of Analytical Chemistry, John Wiley \&Sons LTD.Chichester, 10881-10882.

David A.Z., Mohd E.N., Azlan A. and Chan Y.M. (2013). The Trans fatty acid content in milk and its associate with material diet among lactating mothers in Malaysia. Pg. 46-50.

Dixon D. and Jeena G. (2017).Research article on comparison of different solvent for phytochemical extraction potential from Datura metel plant leaves. International Journal of Biological Chemistry.11:17-22.

Emmanuel Recotti and Garret Fitgerald (2011). Atherosclerosis, thrombosis and inflammation. Vascular biology 31(5): 986-1000.

Environmental Protection Agency USA (2016). Aggregate computational Toxicology Response (ACTOR). Http:/actor.epa.gov/actor/faces/ACTORHOME

European Chemical News (2003). Epichlorohydrin. National Library of Medicine 79(12) 1719.

EU Food improvement Agent (2012). Adopting the list of flavoring substance by European parliament and of the council and repeating commission regulation (EC). NO 1565/2000.

Friedman M., HenikaP. R and Mandwell R. E (2002). Bacterial activity of plants essential oil and some of the isolated constituent against Camphylobacter jeguni, Eschericia Coli, Listeria monocytogens and Salmonella enterica. Journal of Food Protein.65:1545-1560.

Gamze Guelu, and Serkan Seli (2021). Antioxidant activity in Olive oil. Olives and Olive Oil in Health and Diesaes Prevention (2nd Edition).pg. 245-250.

Ganesh.S. Radha.R and Jayshree.N. (2015).A review on phytochemical and pharmacological status of Datura Fastuosalinn. International Journal of Multidisciplinary Research and Developmant 2(4):602-605.

Hameed I. H., Altameme H. J and Didan S. A (2016).Artemisia annua: Biochemical products analysis of methanol aerial parts extract and anti-microbial capacity. Research Journal of $\quad$ Pharmaceutical, Biological and chemical $\quad$ science. 7(2):1843-1868. 
Hayakwa, I,Shioya R., Agatsuma T., Funekwa H., Naneto S., Sugano Y.,(2004). 4-hydroxymethyl-6- phenylbenzofuran-2- carboxylic acid ethyl esters derivatives as potent antitumour agent. Bio org. Med. Chem.Letter. 14: 455- 458

Hossain M.A. and Nagooru M.k. (2011). Biochemical profiling and total flavonoid content of leaves crude extract of endemic medicinal plant amydaline terminal $\mathrm{L}$. Kunth. Pharmacognosy Journal 3(24), 25-29.

Huang P.C., Tien C.J. Sun Y.M and Lee. C.C. (2008). Occurance of phythalates in sediment and biota. Relationship to aquatic factor and biota-sediment accumulation factor. Chemsphere .vol 73 nos 4. pp 539-544.

Ilondu E. M and Lemy E.G. (2018).Studies on the diversity of snake repellant plants within some communities in Delta State Nigeria. International Journal of plant, animals and Environmental Science 8(1):16-24.

Indra R., Bachheti R. K., Joshi A and Pnday D. P. (2013). Phytochemical properties and elemental analysis of some non-cultivated seed oils collected from Garwal Region,Uttarkhand (India) .JICRGG. 5(1):232-236

International Union of Pure and Applied Chemistry (2014). Nomenclature of organic Chemistry. IUPAC Recommendation and Preferred Names the Royal Society of Chemistry pp.724-729.

Jamdhade M.S., Survase S. A., Kare M. A and Bhuktar A.S. (2010). Phytochemical investigation of extracts and the solvent fractionates of the aqueous extracts of Eichhorniacrassipes. Journal Pharmacology Research 4(5):1405-1406.

J.Elks (2014). The dictionary of drugs: chemical data: chemical data, structure and bibliographies. Springer pp.162-ISBN: 978-1-4757-2085-3.

Jesus Valdes-Martinez and Ruben Alfredo Toscano. (2007). 2-methy-3- thiosemicarbazide.

Acta Crystallographic section E. Structure Report Online 63(10): 2.

Jian Zhang, Yangun Gong and Ying Yu (2010). PGF2 receptors: a promising theraupeutic target for cardiovascular disease. Frontiers in Pharmacology. 23:24- 26.

Julie marves (2020). Lorazepam (Atwan). Everyday Health. Pp.23-25.

Kadhim M. J., Soasa A. A and Hameed I.H. (2016). Evaluation of anti-bacterial activity and bioactive chemical analysis of Ocimum basilicum using Fourier Transform Infrared (FTIR) and Gas Chromatography-Mass Spectrometry (GC-MS) techniques.

International Journal of Pharmacognosy and Phytochemical Research .8(6):127-146.

Kent Carlson and Leslie E. Patton (2010). Toxicity review of dissoctyl phthalate (DIOP). United State Consumer Product Safety Commission pg. 1-16.

Khan Z. S and Nasreen S. (2010).Phytochemical analysis, antifungal activities and mode action of methanol extract from plants against pathogen. Journal Agricultural Technology. pg. 274-275.

Lalthanpuii P.B. Zarzokimi and Kholhing L.A. Chandama. (2019) Chemical Profiling, antibacterial and antiparasitic studies of imperata cylinderica. Journal of Applied Pharm. Science. 9(12): 117-121.

Lewis Noble W., Raymond Tietz F., Yun Shik K and Burckhalter J.H. (2012). Antimicrobial agents, Synthesis of Amopyroquine. Journal of Pharmaceutical Science. Volume 52, issue 6. Pp600-61

Love Junweei, Wanxi Peng (2014). Molecular and function of rosewood. Pterocarpus canbodianus, Arabic Journal of Chemistry Volume 11, issue 6. Pg763-770.

Malathi L.K., Anand Anbarasu and Sudha Ramiah. (2016). Medical and biological computional laboratory, school of bioscience and technology. Vit university Vellore India. Indian Journal of Pharmaceutical Science. 78(6) 780-788. 
Mathai, K. (2010). Nutrition in adult. Kruse's Food, Nutrition and Diet Therapy. $10^{\text {th }}$ edition, pg 24-27.

Monir K. M and Munan S.M. (2012). Review on Datura metel a potential medicinal plants. Global Journal of Research on Medicinal Plant and Indigenous Medicine .1(4):123-132.

Muthulakshmi A., Margret J and Mohan V (2012). GC-MS analysis of bioactive components of Ferniale Phantumcorrea (Rutaceae). Applied Pharmac. Sci. (2):69-74.

Nair A., Abrahamsson B., Barend D., Groot D., KoppS., Polli J., Shah V and Dressman J. (2012). Biowaiver monographs for immediate release solid oral dosage Amodiaquine hydrochloromate. Journal of Pharmaceutical Science. forms. 4390-401.

National centre for biotechnology information (2021). Pubchem compound summary .https:/pubchem.ncbi.nlm.nih.gov/compound/10071499.

Okenwa Igwe and Okwunodulum Felicia (2014). Investigationn of Bioactive phytochemical compunds from the chloroform extract of the leave of phyllantus amaraus by GC-MS Technique. International Journal of Chemistry and Pharmaceutical Science vol 2(1): 554-560.

Pillai A.D., Rathod P.D., Xavier F.P, Pad H, Sudarasanam V and Vasu. K.K. (2005). Tetra substituted thiophene as anti-inflammatory agents: exploitation of analoguebased drug design. Bioorganic Med.Chem.13: 6685-6695.

Robert A. Kanaly and Natsuko Hamamura (2018). 9, 10-phenanthrenedione biodegradation by soil bacteria and identification of transformation product. Chemospehre 92(11) 1442-1449.

Ryszard Amarawicz and Michael Janiak (2019). Natural phenolic found in fruits and vegetables .Encyclopedia of Food Chemistry pg. 458-460.

Saku O., Saki M., Kurokwara K., Ikeda T., Takizawa and Uesaka N (2010). Synthesis study on selection adenosine A2A receptor antagonists: synthesis and structure activity relationship of novel benzofuran derivatives. Bioorganic and Medicinal Chemistry Letter Vol. 20. Nos 3 pp 1090-1093.

Sood P. Modgil R., Sood M and Chuhan P (2012). Anti-nutrient profile of different hemopoduim cultivers leaves. Annual Food Science and Technology 13(1) 68-74

Sundaramoorthy Sanutha, Mani Deepa, Nagaraj Sugitha, Sathiavelu Mythili and Arunachalem Sathiavelu (2014). Antioxidant activity and phytochemical Analysis of

Datura metel. International Journal of Drug Development and Research 254-260.

Tania R.D. and Pedro F. O (2018). Phenolic compunds very abundant in tea, coca, and berries. Encyclopedia of Reproduction pg. 458-464.

Toxic substance control Act (TSCA). Chemical substance Inventory: user guide by United State, Environmental Protection Agency. Office of Toxic Substance.pp25- 30.

Van Thuyne W., Van Eenno P., Mikulcikora P., Deventus K and Delbeke F (2005). Detection of androst-4-ene, 3, 6, 17-trione. And its metabolite in Urine by Gas Chromatography. 19(9)689-95.

Vasudevan Aparna (2012). Anti-inflammatory, property of n-hexadecanoic acid: structural evidence and kinetic assessment. Chemical Biology and Drug.30: 25- 29.

Visveshwari M., Subbaiyiyan B and Thagapandian V. (2017). Datura metel is deleterious to the visual cortex of adult Wister Rats. Advances in Applied Science Research 3(2):944-949 
African Journal of Biology and Medical Research

ISSN: $2689-534 \mathrm{X}$

Volume 5, Issue 1, 2022 (pp. 30-52)

www.abjournals.org

Yang B., GuoR., Lilin Y., Wang C., Shu Z., Wang Z., Zhang J., and Xia Y. (2014). Anolides from the leaves of Datura metel and their inhibitory effect on nitric oxide production. Molecules. 19:4548 - 4559.

Zingg J.M., Han S.N., Pang E., Meydani M., Maydani S.N and Azzi A. (2013). In vivo regulation of gene transcription by alpha- and gamma tocopherol in murine $\mathrm{T}$. Lymphocytes. Archives of Biochemistry and Biophysics 538-119. 